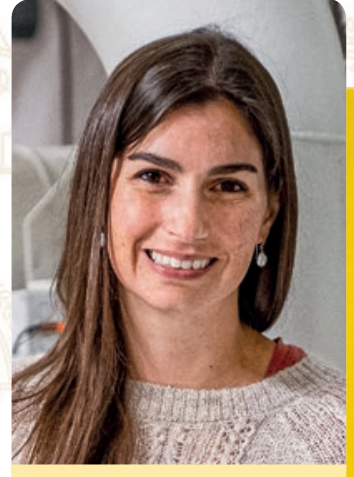

Denise Mitrano

(๔ SNF / Cornelia Vinzens)

\section{Marie Heim-Vögtlin-Preis} 2021

Denise Mitrano, Geochemikerin an der ETH Zürich, erhält vom Schweizerischen Nationalfonds (SNF) den Marie Heim-VögtlinPreis 2021 (mit CHF 25000 dotiert). Die gebürtige US-Amerikanerin entwickelte ein Verfahren, mit dem sich Mikro- und Nanoplastik in Gewässern, Böden und sogar Organismen nachverfolgen lässt. Bisher war es kaum möglich, den Verbreitungswegen dieser bis zu wenigen Millionstel Millimeter kleinen Partikel auf die Spur zu kommen. Finanziert wurde diese Arbeit durch einen Ambizione-Beitrag im Rahmen der Nachwuchsförderung des SNF. Seit 2020 leitet Denise Mitrano als Assistenzprofessorin mit einem Eccellenza Fellowship des SNF ihre eigene Forschungsgruppe an der ETH Zürich.

\section{Prix FORESO 2021}

Emil Scolari, maître d'enseignement HES, a obtenu le Prix FORESO 2021 qui récompense son travail de Master en Sciences Infirmières de la Faculté

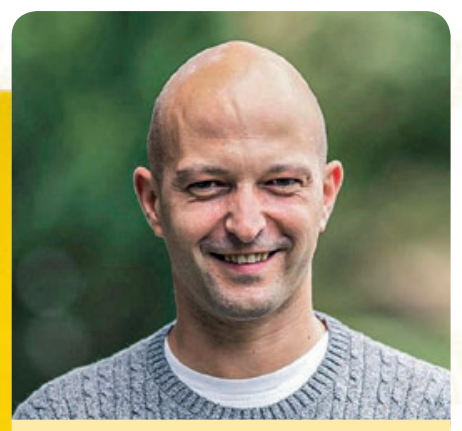
Emil Scolari (๑ HESAV) de biologie et de médecine de Lausanne. Réalisée au CHUV, où il occupait le poste d'infirmier clinicien spécialisé aux soins intensifs, cette étude s'intitule «Utilisation du SCAR par les infirmiers de soins intensifs lors des appels téléphoniques au médecin: Une étude observationnelle». Elle vise à décrire la qualité de l'utilisation de l'outil de communication SCAR et l'influence des différentes formations et de l'expérience professionnelle des infirmiers et infirmières sur la qualité de son utilisation.

La Fondation pour la recherche en soins (FORESO) a pour but de promouvoir la recherche dans les différentes professions non médicales de la santé, en particulier les soins infirmiers.

\section{Kenneth Johnson Award 2021}

Prof. Victor Valderrabano wurde in Charlotte, North Carolina, USA, für seine langjährige Forschung, Lehre und Innovation im Bereich der Arthrose des Sprunggelenks und Sprunggelenksprothese mit dem «Kenneth A. Johnson International Speaker Award» der American Orthopedic Foot and Ankle Society (AOFAS) ausgezeichnet. Der Chefarzt des SWISS ORTHO CENTER bei Swiss Medical Network und Professor für Orthopädische Chirurgie an der Universität Basel ist ein international anerkannter Chirurg und Forscher.

\section{Gesundheitsnetz 2025 zeichnet zwei innovative Projekte aus}

Das Gesundheitsnetz 2025 hat zum zehnten Mal innovative Projekte in der Zürcher Gesundheitsversorgung ausgezeichnet: zum einen das Projekt «Visit - Spital Zollikerberg Zuhause», zum anderen den digitalen «NoA Coach» der Suchtfachstelle Zürich. Das Preisgeld von je CHF 10000 stiftet die Gesundheitsdirektion des Kantons Zürich. «Visit - Spital Zollikerberg Zuhause» bietet Menschen ab Spätherbst 2021 mit bestimmten hospitalisationsbedürftigen Erkrankungen eine Behandlung und Betreuung im häuslichen Umfeld, die aus qualitativer Sicht einem stationären Spitalaufenthalt entspricht. Das Projekt wurde von einem interprofessionellen Team des Spitals Zollikerberg und des Instituts Neumünster entwickelt. «NoA Coach» erzielt eine optimierte Behandlung von Alkoholproblemen durch digital unterstütztes Selbstmanagement. 